\title{
Fermat collocation method for the solutions of nonlinear system second order boundary value problems
}

\author{
Salih Yalcinbas, Kubra Erdem Bicer and Dilek Tastekin \\ Department of Mathematics Celal Bayar University, Manisa-Turkey
}

Received: 13 October 2015, Revised: 21 October 2015, Accepted: 23 November 2015

Published online: 17 January 2016.

\begin{abstract}
In this study, a numerical approach is proposed to obtain approximate solutions of nonlinear system of second order boundary value problem. This technique is essentially based on the truncated Fermat series and its matrix representations with collocation points. Using the matrix method, we reduce the problem system of nonlinear algebraic equations. Numerical examples are also given to demonstrate the validity and applicability of the presented technique. The method is easy to implement and produces accurate results.
\end{abstract}

Keywords: Boundary value problems, matrix methods, Fermat polynomials, nonlinear systems.

\section{Introduction}

Differential equation systems has been one of the essential tools for various areas of scientific fields such as biology, ecology, chemistry, medicine, physics and engineering. Many scientific applications lead to higher order systems of ordinary differential equations, but it is often difficult or impossible to obtain explicit solutions to higher order or coupled systems of nonlinear ordinary differential equations. The method we shall discuss here is aimed primarily at discovering something of the character of the solutions. For simplicity, we shall concentrate on second order systems; the method may readily be generalized to higher order systems.

In this paper, we will consider the following nonlinear system of second order differential equations:

$$
\left.\begin{array}{l}
a_{1}(t) y_{1}^{\prime \prime}+a_{2}(t) y_{1}^{\prime}+a_{3}(t) y_{1}+a_{4}(t) y_{2}^{\prime \prime}+a_{5}(t) y_{2}{ }^{\prime}+a_{6}(t) y_{2}+N_{1}\left(y_{1}, y_{2}\right)=g_{1}(t) \\
b_{1}(t) y_{1}{ }^{\prime \prime}+b_{2}(t) y_{1}^{\prime}+b_{3}(t) y_{1}+b_{4}(t) y_{2}^{\prime \prime}+b_{5}(t) y_{2}{ }^{\prime}+b_{6}(t) y_{2}+N_{2}\left(y_{1}, y_{2}\right)=g_{2}(t)
\end{array}\right\} 0 \leq t \leq 1
$$

with boundary conditions

$$
\left.\begin{array}{l}
y_{1}(0)=y_{1}(1)=0 \\
y_{2}(0)=y_{2}(1)=0
\end{array}\right\}
$$

where $0 \leq t \leq 1, N_{1}$ and $N_{2}$ are nonlinear functions of $y_{1}$ and $y_{2}$. Also $a_{i}(t), b_{i}(t)$ for $i=1, \ldots, 6$ are given continuous functions and $g_{1}$ and $g_{2}$ are known functions.

Geng and Cui [1] has represented the analytical solution of problem (1)-(2) in the form of series in the reproducing kernel space under the assumption that the solution to problem (1)-(2). Lu [2], proposed a variational iteration method, Dehghan and Saadatmandi [3] presented a numerical method based on Sinc-collocation method, Saadatmandi and Farsangi [4] solved this problem by using the Chebyshev finite difference method. Dehghan and Lakestani [5] suggested 
a numerical method base on the cubic B-spline scaling functions to find the solutions of the system. Also, Saadatmandi [6] has tried to solve problem (1)-(2) by using He's homotopy perturbation method. The existence and uniqueness of solutions of second-order systems have been discussed, including the approximation of solutions via finite difference method [7-14].

Since the beginning of 1994, Taylor, Chebyshev, Legendre, Laguerre, Bernstein and Bessel collocation and matrix methods have been used by many authors [15-20] to solve differential, difference, integral, integro-differential, delay differential equations and their systems. Taştekin and Yalçınbaş [21] have used Fermat collocation method to solve first order nonlinear differential equations. Also Yalçınbaş and Taştekin [22] have considered the solution of second order nonlinear differential equations by using Fermat collocation method.

In the present work, we want to find the approximate solutions of the system (1) with boundary conditions (2) in the truncated Fermat series form

$$
y_{i, N}(t)=\sum_{n=0}^{N} y_{i, n} F_{n}(t), i=1,2,0 \leq t \leq b,
$$

where $y_{i, n},(n=0,1, \ldots, N, i=1,2)$ are unknown coefficients to be determined.

The organization of this paper is as follows: In the next section we describe the matrix representations of each term in the system (1) and (2). In Section 3, we find the fundamental matrix relation of this system. In Section 4, the Fermat collocation method is performed. In Section 5, the accuracy of solution is given and in Section 6, some computational results are given to clarify the method. Section 7 ends this paper with a brief conclusion.

\section{Fundamental relations}

Let us consider the nonlinear system in the form (1) and find the matrix representations of each term in the system. First we convert the solution defined by (3) and its derivatives, for $n=0,1, \ldots, N$ to the following matrix forms:

$$
\begin{aligned}
& y_{i}(t)=F(t) Y_{i}, i=1,2,[20,21] \\
& y_{i}^{(n)}(t)=F(t) D^{n} Y_{i}, i=1,2
\end{aligned}
$$

where,

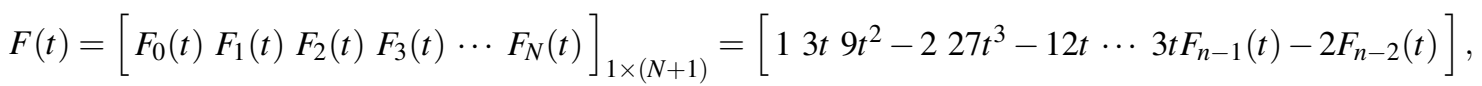

$$
\begin{aligned}
& D=\left[\begin{array}{llllllllll}
0 & 3 & 0 & 6 & 0 & 12 & 0 & 24 & 0 & \cdots \\
0 & 0 & 6 & 0 & 12 & 0 & 24 & 0 & 48 & \cdots \\
0 & 0 & 0 & 9 & 0 & 18 & 0 & 36 & 0 & \cdots \\
0 & 0 & 0 & 0 & 12 & 0 & 24 & 0 & 48 & \cdots \\
0 & 0 & 0 & 0 & 0 & 15 & 0 & 30 & 0 & \cdots \\
0 & 0 & 0 & 0 & 0 & 0 & 18 & 0 & 36 & \cdots \\
0 & 0 & 0 & 0 & 0 & 0 & 0 & 21 & 0 & \cdots \\
0 & 0 & 0 & 0 & 0 & 0 & 0 & 0 & 24 & \cdots \\
\vdots & \vdots & \vdots & \vdots & \vdots & \vdots & \vdots & \vdots & \vdots & \ddots \\
0 & 0 & 0 & 0 & 0 & 0 & 0 & 0 & 0 & \cdots
\end{array}\right]_{(N+1) \times(N+1)}, Y_{i}=\left[\begin{array}{c}
y_{i, 0} \\
y_{i, 1} \\
y_{i, 2} \\
\vdots \\
y_{i, N}
\end{array}\right]_{(N+1) \times 1}
\end{aligned}
$$


Nonlinear part of the system (1), $N_{i}\left(y_{1}, y_{2}\right), i=1,2$ can be found as $y_{i}{ }^{2}(t)$ or $y_{i}(t) y_{j}(t), i \neq j, i=j=1,2$. Also, we can write the matrix form of these nonlinear expressions

$$
\begin{aligned}
& y_{i}^{2}(t)=F(t) F^{*}(t) \bar{Y}_{i, i} \\
& y_{i}(t) y_{j}(t)=F(t) F^{*}(t) \bar{Y}_{i, j} \\
& y_{j}(t) y_{i}(t)=F(t) F^{*}(t) \bar{Y}_{j, i}
\end{aligned}
$$

where

$$
F^{*}(t)=\left[\begin{array}{cccc}
F(t) & 0 & \cdots & 0 \\
0 & F(t) & \cdots & 0 \\
\vdots & \vdots & \ddots & \vdots \\
0 & 0 & \cdots & F(t)
\end{array}\right]_{(N+1) \times(N+1)^{2}}
$$

and

$$
\bar{Y}_{i, i}=\left[\begin{array}{llll}
y_{i, 0} Y_{i} & y_{i, 1} Y_{i} & \cdots & y_{i, N} Y_{i}
\end{array}\right]_{(N+1)^{2} \times 1}^{T}, \quad \bar{Y}_{i, j}=\left[\begin{array}{llll}
y_{j, 0} Y_{i} & y_{j, 1} Y_{i} & \cdots & y_{j, N} Y_{i}
\end{array}\right]_{(N+1)^{2} \times 1}^{T} .
$$

\section{Fundamental matrix equations for system}

We are now ready to construct the fundamental matrix equations for the nonlinear system of second order boundary value problem (1). For this purpose, substituting the matrix relations (4)-(8) into system (1) and simplifying, we obtain the system of matrix equations:

$$
\left.\begin{array}{c}
a_{1}(t) F(t) D^{2} Y_{1}+a_{2}(t) F(t) D Y_{1}+a_{3}(t) F(t) Y_{1}+a_{4}(t) F(t) D^{2} Y_{2}+a_{5}(t) F(t) D Y_{2} \\
+a_{6}(t) F(t) Y_{2}+N_{1}(t) \bar{Y}_{i, j}=g_{1}(t) \\
b_{1}(t) F(t) D^{2} Y_{1}+b_{2}(t) F(t) D Y_{1}+b_{3}(t) F(t) Y_{1}+b_{4}(t) F(t) D^{2} Y_{2}+b_{5}(t) F(t) D Y_{2} \\
+b_{6}(t) F(t) Y_{2}+N_{2}(t) \bar{Y}_{j, i}=g_{2}(t)
\end{array}\right\}
$$

Therefore, we can write the matrix representation of the system (9) in the form

$$
\begin{aligned}
& \underbrace{\left[a_{1}(t) F(t) D^{2}+a_{2}(t) F(t) D+a_{3}(t) F(t)\right]}_{A_{1}(t)} Y_{1}+\underbrace{\left[a_{4}(t) F(t) D^{2}+a_{5}(t) F(t) D+a_{6}(t) F(t)\right]}_{A_{2}(t)} Y_{2}+N_{1}(t) \bar{Y}_{i, j}=g_{1}(t)\} \\
& \underbrace{\left[b_{1}(t) F(t) D^{2}+b_{2}(t) F(t) D+b_{3}(t) F(t)\right]}_{B_{1}(t)} Y_{1}+\underbrace{\left[b_{4}(t) F(t) D^{2}+b_{5}(t) F(t) D+b_{6}(t) F(t)\right]}_{B_{2}(t)} Y_{2}+N_{2}(t) \bar{Y}_{j, i}=g_{2}(t)\} \\
& \left.\begin{array}{l}
A_{1}(t) Y_{1}+A_{2}(t) Y_{2}+N_{1}(t) \bar{Y}_{i, j}=g_{1}(t) \\
B_{1}(t) Y_{1}+B_{2}(t) Y_{2}+N_{2}(t) \bar{Y}_{j, i}=g_{2}(t)
\end{array}\right\}
\end{aligned}
$$

where

$$
\begin{aligned}
& A_{1}(t)=a_{1}(t) F(t) D^{2}+a_{2}(t) F(t) D+a_{3}(t) F(t), \\
& A_{2}(t)=a_{4}(t) F(t) D^{2}+a_{5}(t) F(t) D+a_{6}(t) F(t),
\end{aligned}
$$


and

$$
\begin{aligned}
& B_{1}(t)=b_{1}(t) F(t) D^{2}+b_{2}(t) F(t) D+b_{3}(t) F(t), \\
& B_{2}(t)=b_{4}(t) F(t) D^{2}+b_{5}(t) F(t) D+b_{6}(t) F(t) .
\end{aligned}
$$

Consequently, the fundamental matrix equations of the system (10) can be written in the following compact form

$$
P(t) Y+N(t) \bar{Y}=G(t)
$$

where

$$
\begin{aligned}
& P(t)=\left[\begin{array}{ll}
A_{1}(t) & A_{2}(t) \\
B_{1}(t) & B_{2}(t)
\end{array}\right]_{2 \times 2(N+1)}, Y=\left[\begin{array}{l}
Y_{1} \\
Y_{2}
\end{array}\right]_{2(N+1) \times 1}, \bar{Y}=\left[\begin{array}{l}
\bar{Y}_{i, j} \\
\bar{Y}_{j, i}
\end{array}\right]_{2(N+1)^{2} \times 1}, \\
& N(t)=\left[\begin{array}{cc}
N_{1}(t) & 0 \\
0 & N_{2}(t)
\end{array}\right]_{2 \times 2(N+1)^{2}}, G(t)=\left[\begin{array}{l}
g_{1}(t) \\
g_{2}(t)
\end{array}\right]_{2 \times 1} .
\end{aligned}
$$

\section{Fermat collocation method}

In this section, by substituting the collocation points defined by

$$
t_{s}=\frac{b}{N} s, s=0,1, \ldots, N
$$

into the fundamental matrix equation (11), we obtain the new system

$$
P\left(t_{s}\right) Y+N\left(t_{s}\right) \bar{Y}=G\left(t_{s}\right)
$$

and therefore, the new fundamental matrix equation

$$
W Y^{*}+V \bar{Y}^{*}=G^{*}
$$

where

$$
\begin{aligned}
W & =\left[\begin{array}{cccc}
P\left(t_{0}\right) & 0 & \cdots & 0 \\
0 & P\left(t_{1}\right) & \cdots & 0 \\
\vdots & \vdots & \ddots & \vdots \\
0 & 0 & \cdots & P\left(t_{N}\right)
\end{array}\right]_{2(N+1) \times 2(N+1)^{2}}, \quad V=\left[\begin{array}{cccc}
N\left(t_{0}\right) & 0 & \cdots & 0 \\
0 & N\left(t_{1}\right) & \cdots & 0 \\
\vdots & \vdots & \ddots & \vdots \\
0 & 0 & \cdots & N\left(t_{N}\right)
\end{array}\right]_{2(N+1) \times 2(N+1)^{3}}, \\
Y^{*} & =\left[\begin{array}{c}
Y \\
Y \\
\vdots \\
Y
\end{array}\right]_{2(N+1)^{2} \times 1} \quad, \quad \bar{Y}^{*}=\left[\begin{array}{c}
\bar{Y} \\
\bar{Y} \\
\vdots \\
\bar{Y}
\end{array}\right]_{2(N+1)^{3} \times 1}, \quad G^{*}=\left[\begin{array}{c}
G\left(t_{0}\right) \\
G\left(t_{1}\right) \\
\vdots \\
G\left(t_{N}\right)
\end{array}\right] .
\end{aligned}
$$

To find matrix representation of boundary conditions given with (2), by using Eq. 4 we can write row matrices as

$$
F(0) Y_{1}=0, \quad F(0) Y_{2}=0 \quad \text { and } \quad F(1) Y_{1}=0, \quad F(1) Y_{2}=0 .
$$


Thus, we obtain the matrix forms of the conditions, respectively,

$$
U_{0} Y=0 \text { and } U_{1} Y=0
$$

where

$$
U_{0}=\left[\begin{array}{cc}
F(0) & 0 \\
0 & F(0)
\end{array}\right]_{2 \times 2(N+1)}, \quad U_{1}=\left[\begin{array}{cc}
F(1) & 0 \\
0 & F(1)
\end{array}\right]_{2 \times 2(N+1)}, \quad Y=\left[\begin{array}{l}
Y_{1} \\
Y_{2}
\end{array}\right]_{2(N+1) \times 1} .
$$

To obtain the approximate solution of Eq. (1) with boundary conditions (2) in the terms of Fermat polynomials, by replacing the row matrix (14) by the last row of the matrix (13),we obtain the required augmented matrix:

$$
\left[\tilde{W}, \tilde{V} ; \tilde{G}^{*}\right]=\left[\begin{array}{lllllllllllll}
P\left(t_{0}\right) & 0 & 0 & \cdots & 0 & 0 & , N\left(t_{0}\right) & 0 & 0 & \cdots & 0 & 0 & ; G\left(t_{0}\right) \\
0 & P\left(t_{1}\right) & 0 & \cdots & 0 & 0 & , 0 & N\left(t_{1}\right) & 0 & \cdots & 0 & 0 & ; G\left(t_{1}\right) \\
\vdots & \vdots & \vdots & \ldots & \vdots & \vdots & , \vdots & \vdots & \vdots & \cdots & \vdots & \vdots & ; \\
0 & 0 & 0 & \cdots & U_{0} & 0 & , 0 & 0 & 0 & \cdots & 0 & 0 & ; 0 \\
0 & 0 & 0 & \cdots & 0 & U_{1} & , 0 & 0 & 0 & \cdots & 0 & 0 & ; 0
\end{array}\right]
$$

and the corresponding matrix equation

$$
\tilde{W} Y^{*}+\tilde{V} \bar{Y}^{*}=\tilde{G}^{*}
$$

where

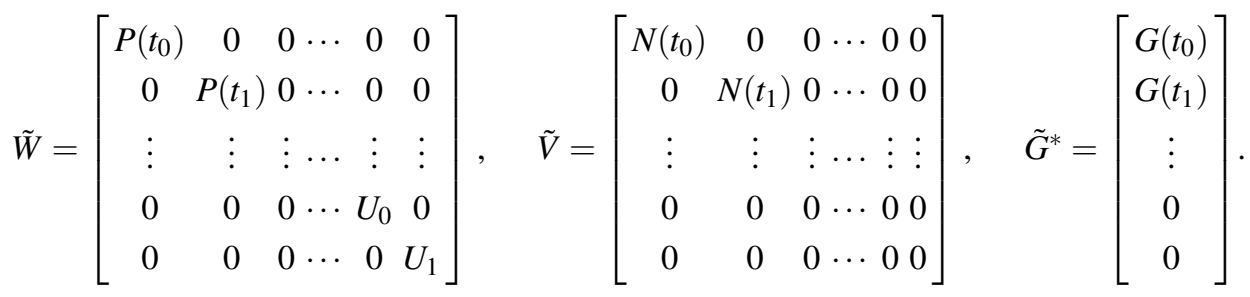

The fundamental matrix equation of the system (1) under boundary conditions (2) corresponds to a system of $2(N+1)$ nonlinear algebraic equations with the unknown coefficients $y_{1, n}$ and $y_{2, n},(n=0,1, \ldots, N)$.

Finally, the unknown coefficients are computed by solving this system and they are substituted in Eq. 3. Hence, the Fermat polynomial solutions

$$
y_{i, N}(t)=\sum_{n=0}^{N} y_{i, n} F_{n}(t), \quad i=1,2
$$

can be obtained.

\section{Accuracy of solutions}

We can easily check the accuracy of the above solutions. Since truncated Fermat series (3) is the approximate solution of system (1), when the function $y_{i, N}(t), i=1,2$ and its derivatives are substituted in system (1), the resulting equation must be satisfied approximately; that is, for $t=t_{q} \in[0,1], q=0,1,2, \ldots$,

$$
E_{1, N}\left(t_{q}\right)=\left|\begin{array}{l}
a_{1}\left(t_{q}\right) y_{1, N}{ }^{\prime \prime}+a_{2}\left(t_{q}\right) y_{1, N}{ }^{\prime}+a_{3}\left(t_{q}\right) y_{1, N}+a_{4}\left(t_{q}\right) y_{2, N}{ }^{\prime \prime}+ \\
a_{5}\left(t_{q}\right) y_{2, N}{ }^{\prime}+a_{6}\left(t_{q}\right) y_{2, N}+N_{1}\left(y_{1}\left(t_{q}\right), y_{2}\left(t_{q}\right)\right)-g_{1}\left(t_{q}\right)
\end{array}\right| \cong 0
$$




$$
E_{2, N}\left(t_{q}\right)=\left|\begin{array}{l}
b_{1}\left(t_{q}\right) y_{1, N}{ }^{\prime \prime}+b_{2}\left(t_{q}\right) y_{1, N}+b_{3}\left(t_{q}\right) y_{1, N}+b_{4}\left(t_{q}\right) y_{2, N}{ }^{\prime \prime}+ \\
b_{5}\left(t_{q}\right) y_{2, N}{ }^{\prime}+b_{6}\left(t_{q}\right) y_{2, N}+N_{2}\left(y_{1}\left(t_{q}\right), y_{2}\left(t_{q}\right)\right)-g_{2}\left(t_{q}\right)
\end{array}\right| \cong 0,
$$

and $E_{i, N}\left(t_{q}\right) \leq 10^{-k_{q}}, i=1,2$ ( $k_{q}$ positive integer). If $\max 10^{-k_{q}}=10^{-k}\left(k_{q}\right.$ positive integer) is prescribed, then the truncation limit $N$ is increased until the difference $E_{i, N}\left(t_{q}\right)$ at each of the points becomes smaller than the prescribed $10^{-k}[22]$.

\section{Numerical examples}

In this section, numerical examples are given to illustrate the accuracy and efficiency of the presented method.

Example 1. Let us first consider the nonlinear system of second order boundary value problems [23],

$$
\left.\begin{array}{c}
y_{1}^{\prime \prime}-t y_{2}^{\prime}+t y_{1}=t^{3}-2 t^{2}+6 t \\
y_{2}^{\prime \prime}+t y_{1}^{\prime}+y_{1} y_{2}=t^{5}-t^{4}+2 t^{3}+t^{2}-t+2
\end{array}\right\}, \quad 0 \leq t \leq 1
$$

with boundary condition $y_{1}(0)=y_{1}(1)=0$ and $y_{2}(0)=y_{2}(1)=0$. The exact solutions of this problem are $y_{1}(t)=t^{3}-t$, $y_{2}=t^{2}-t$. Now, let us apply the procedure in Section 4 to obtain this approximate solution. Firstly, we note that

$$
\begin{aligned}
& a_{1}(t)=1, \quad a_{3}(t)=t, \quad a_{5}(t)=-t, a_{2}(t)=a_{4}(t)=a_{6}(t)=0, b_{2}(t)=t, \quad b_{4}(t)=1, \\
& b_{1}(t)=b_{3}(t)=b_{5}(t)=b_{6}(t)=0, N_{1}\left(y_{1}, y_{2}\right)=0, \quad N_{2}\left(y_{1}, y_{2}\right)=1, g_{1}(t)=t^{3}-2 t^{2}+6 t, \\
& g_{2}(t)=t^{5}-t^{4}+2 t^{3}+t^{2}-t+2 .
\end{aligned}
$$

The set of collocation points for $N=3$ is computed as $\left\{t_{0}=0, t_{1}=\frac{1}{3}, t_{2}=\frac{2}{3}, t_{1}=1\right\}$ and the fundamental matrix equation of the problem from Eq. 10 is

$$
\left.\begin{array}{c}
{\left[\mathbf{F}(t) \mathbf{D}^{2}+\mathbf{F}(t)\right] \mathbf{Y}_{1}-t \mathbf{F}(t) \mathbf{D} \mathbf{Y}_{2}=t^{3}-2 t^{2}+6 t} \\
(t) \mathbf{D} \mathbf{Y}_{1}+\mathbf{F}(t) \mathbf{D}^{2} \mathbf{Y}_{2}+\overline{\mathbf{Y}}_{j, i}=t^{5}-t^{4}+2 t^{3}+t^{2}-t+2
\end{array}\right\}
$$

We can find the compact form of this system from Eq. 11 as

$$
P(t) Y+N(t) \bar{Y}=G(t)
$$

where

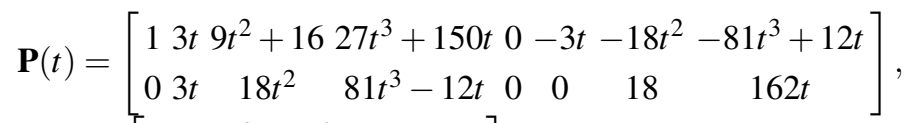

$$
\begin{aligned}
& \mathbf{G}(t)=\left[\begin{array}{c}
t^{3}-2 t^{2}+6 t \\
t^{5}-t^{4}+2 t^{3}+t^{2}-t+2
\end{array}\right] .
\end{aligned}
$$

The fundamental matrix equation

$$
W Y^{*}+V \bar{Y}^{*}=G^{*}
$$

where 


$$
\begin{gathered}
\mathbf{W}=\left[\begin{array}{cccc}
\mathbf{P}(0) & 0 & 0 & 0 \\
0 & \mathbf{P}\left(\frac{1}{2}\right) & 0 & 0 \\
0 & 0 & \mathbf{P}\left(\frac{2}{3}\right) & 0 \\
0 & 0 & 0 & \mathbf{P}(1)
\end{array}\right], \mathbf{V}=\left[\begin{array}{cccc}
\mathbf{N}(0) & 0 & 0 & 0 \\
0 & \mathbf{N}\left(\frac{1}{2}\right) & 0 & 0 \\
0 & 0 & \mathbf{N}\left(\frac{2}{3}\right) & 0 \\
0 & 0 & 0 & \mathbf{N}(1)
\end{array}\right], \\
\mathbf{G}^{*}=\left[\begin{array}{llll}
\mathbf{G}(0) & \mathbf{G}\left(\frac{1}{3}\right) & \mathbf{G}\left(\frac{2}{3}\right) & \mathbf{G}(1)
\end{array}\right]^{T} .
\end{gathered}
$$

The matrix forms of the conditions are

$$
\mathbf{U}_{0}=\left[\begin{array}{llllllll}
1 & 0 & -2 & 0 & 0 & 0 & 0 & 0 \\
0 & 0 & 0 & 0 & 1 & 0 & -2 & 0
\end{array}\right], \mathbf{U}_{1}=\left[\begin{array}{llllllll}
1 & 3 & 7 & 15 & 0 & 0 & 0 & 0 \\
0 & 0 & 0 & 0 & 1 & 3 & 7 & 15
\end{array}\right] .
$$

The augmented matrix for this fundamental matrix equation is calculated as

$$
\left[\tilde{\mathbf{W}}, \tilde{\mathbf{V}} ; \tilde{\mathbf{G}}^{*}\right]=\left[\begin{array}{lllllllllll}
\mathbf{P}(0) & 0 & 0 & 0 & , & \mathbf{N}(0) & 0 & 0 & 0 & ; & \mathbf{G}(0) \\
0 & \mathbf{P}\left(\frac{1}{3}\right) & 0 & 0 & , & 0 & \mathbf{N}\left(\frac{1}{3}\right) & 0 & 0 & ; & \mathbf{G}\left(\frac{1}{3}\right) \\
0 & 0 & \mathbf{U}_{0} & 0 & , & 0 & 0 & 0 & 0 & ; & 0 \\
0 & 0 & 0 & \mathbf{U}_{1}, & 0 & 0 & 0 & 0 & ; & 0
\end{array}\right]
$$

where

$$
\begin{aligned}
& \mathbf{P}(0)=\left[\begin{array}{llllllll}
1 & 0 & 16 & 0 & 0 & 0 & 0 & 0 \\
0 & 0 & 0 & 0 & 0 & 0 & 18 & 0
\end{array}\right], \mathbf{P}\left(\frac{1}{3}\right)=\left[\begin{array}{llllllll}
1 & 1 & 17 & 51 & 0 & -1 & -2 & 1 \\
0 & 1 & 2 & -1 & 0 & 0 & 18 & 54
\end{array}\right] \\
& \mathbf{N}(0)=\left[\begin{array}{lllllllllllllllll}
\mathbf{0} & & & & & & & & \mathbf{0} & & & & & & & \\
\mathbf{0} & 1 & 0 & -2 & 0 & 0 & 0 & 0 & 0 & -2 & 0 & 4 & 0 & 0 & 0 & 0 & 0
\end{array}\right]_{2 \times 32}, \\
& \mathbf{N}\left(\frac{1}{3}\right)=\left[\begin{array}{llllllllllllllllll}
\mathbf{0} & & & & \mathbf{0} & \mathbf{0} \\
\mathbf{0} & 1 & 1 & -1 & -3 & 1 & 1 & -1 & -3 & -1 & -1 & 1 & 3 & -3 & -3 & 3 & 9
\end{array}\right]_{2 \times 32}, \\
& \mathbf{G}(0)=\left[\begin{array}{l}
0 \\
2
\end{array}\right], \mathbf{G}\left(\frac{1}{3}\right)=\left[\begin{array}{c}
\frac{49}{27} \\
\frac{523}{243}
\end{array}\right] .
\end{aligned}
$$

By solving this system, the Fermat coefficients matrix is gained as

$$
Y_{1}=\left[\begin{array}{llll}
0 & \frac{5}{27} & 0 & \frac{1}{27}
\end{array}\right]^{T} \text { and } Y_{2}=\left[\begin{array}{cccc}
\frac{2}{9} & -\frac{1}{3} & \frac{1}{9} & 0
\end{array}\right]^{T} .
$$

Substituting the elements of these column matrixes into Eq. 4, we obtain the solution set in terms of Fermat polynomials as

$$
y_{1}(t)=t^{3}-t, y_{2}(t)=t^{2}-t
$$

which are the exact solutions.

Example 2. Other example is the nonlinear system

$$
\left.\begin{array}{c}
y^{\prime \prime}+t y_{2}+t y_{1}^{2}=\left(t^{2}-1\right) \sin t-2 \cos t+\left(t^{3}-2 t^{2}+t\right) \sin ^{2} t \\
y^{\prime \prime}{ }_{2}+t y^{\prime}{ }_{1}+y_{2}=\left(-t^{2}+t+2\right) \cos t-t \sin t
\end{array}\right\}, 0 \leq t \leq 1
$$


with the boundary conditions $y_{1}(0)=y_{1}(1)=0$ and $y_{2}(0)=y_{2}(1)=0$. The exact solutions of this problem are $y_{1}(t)=$ $(1-t) \sin t$ and $y_{2}(t)=(t-1) \sin t$. Using the procedure in Section 4, we calculate the approximate solutions $y_{1, N}(t)$ and $y_{2, N}(t)$ for $N=3,6,8$. In Tables 1-2, the exact solutions and approximate solutions obtained by the present method are compared. On the other hand, in Fig. 1-2 and Fig. 3-4, respectively, the approximate solutions for the present method and absolute errors for present method are shown for different values of $N$. Additionally, in Table 3, the accuracy of solutions are stated. These results show that if $N$ increases, than the absolute errors decrease more rapidly.

Table 1: Comparison of the numerical errors for $y_{1}(t)$

\begin{tabular}{|l|l|l|l|l|l|l|l|}
\hline \multirow{2}{*}{$t_{i}$} & \multicolumn{1}{|c|}{$\begin{array}{l}\text { Exact } \\
\text { Solution }\end{array}$} & \multicolumn{2}{l|}{$\begin{array}{l}N=3 \text { Absolute } \\
y_{1,3}\left(t_{i}\right) \text { Error }\end{array}$} & \multicolumn{2}{l|}{$\begin{array}{l}N=6 \text { Absolute } \\
y_{1,6}\left(t_{i}\right) \text { Error }\end{array}$} & \multicolumn{2}{l|}{$\begin{array}{l}\text { Absolute } \\
y_{1,8}\left(t_{i}\right) \text { Error }\end{array}$} \\
\hline 0 & 0 & 0 & 0 & 0 & 0 & 0 & 0 \\
\hline 0.2 & 0.158935 & 0.158672 & $2.632845 \mathrm{E}-4$ & 0.158935 & $4.422558 \mathrm{E}-8$ & 0.158935 & $4.857349 \mathrm{E}-10$ \\
\hline 0.4 & 0.233651 & 0.229351 & $4.299543 \mathrm{E}-3$ & 0.233651 & $2.500261 \mathrm{E}-7$ & 0.233651 & $8.754586 \mathrm{E}-10$ \\
\hline 0.6 & 0.225856 & 0.204031 & $2.182503 \mathrm{E}-2$ & 0.225858 & $1.955790 \mathrm{E}-6$ & 0.225856 & $1.041634 \mathrm{E}-8$ \\
\hline 0.8 & 0.143471 & 0.074707 & $6.876343 \mathrm{E}-2$ & 0.143478 & $7.573873 \mathrm{E}-6$ & 0.143471 & $7.438348 \mathrm{E}-8$ \\
\hline 1 & 0 & 0.16662 & $1.666269 \mathrm{E}-1$ & 0.000001 & $1.207777 \mathrm{E}-5$ & 0.000000 & $1.333333 \mathrm{E}-7$ \\
\hline
\end{tabular}

Table 2: Comparison of the numerical errors for $y_{2}(t)$

\begin{tabular}{|l|l|l|l|l|l|l|l|}
\hline \multirow{2}{*}{$t_{i}$} & $\begin{array}{c}\text { Exact } \\
\text { Solution }\end{array}$ & $\begin{array}{l}N=3 \\
y_{1,3}\left(t_{i}\right)\end{array}$ & Absolute & \multicolumn{2}{l|}{$\begin{array}{l}N=6 \\
\text { Error }\end{array}$} & $\begin{array}{l}\text { Absolute } \\
y_{1,6}\left(t_{i}\right)\end{array}$ & \multicolumn{2}{l|}{$\begin{array}{l}\text { Error } \\
y_{1,8}\left(t_{i}\right)\end{array}$} & \multicolumn{2}{l}{$\begin{array}{l}\text { Absolute } \\
\text { Error }\end{array}$} \\
\hline 0 & 0 & 0 & 0 & 0 & 0 & 0 & 0 \\
\hline 0.2 & -0.158935 & -0.158672 & $2.632845 \mathrm{E}-4$ & -0.158935 & $4.422558 \mathrm{E}-8$ & -0.158935 & $4.857349 \mathrm{E}-10$ \\
\hline 0.4 & -0.233651 & -0.229351 & $4.299543 \mathrm{E}-3$ & -0.233651 & $2.500261 \mathrm{E}-7$ & -0.233651 & $8.754586 \mathrm{E}-10$ \\
\hline 0.6 & -0.225856 & -0.204031 & $2.182503 \mathrm{E}-2$ & -0.225858 & $1.955790 \mathrm{E}-6$ & -0.225856 & $1.041634 \mathrm{E}-8$ \\
\hline 0.8 & -0.143471 & -0.074707 & $6.876343 \mathrm{E}-2$ & -0.143478 & $7.573873 \mathrm{E}-6$ & -0.143471 & $7.438348 \mathrm{E}-8$ \\
\hline 1 & 0 & -0.16662 & $1.666269 \mathrm{E}-1$ & -0.000001 & $1.207777 \mathrm{E}-5$ & -0.000000 & $1.333333 \mathrm{E}-7$ \\
\hline
\end{tabular}

Table 3: Absolute errors of $y_{1}(t)$ and $y_{2}(t)$

\begin{tabular}{|l|l|l|}
\hline$t_{i}$ & \multicolumn{1}{|c|}{$N=8, y_{1,8}\left(t_{i}\right), y_{2,8}\left(t_{i}\right)$} & \multicolumn{1}{|c|}{$N=10, y_{1,10}\left(t_{i}\right), y_{2,10}\left(t_{i}\right)$} \\
\hline 0 & 0 & 0 \\
\hline 0.2 & $4.857349373 \mathrm{E}-10$ & $4.1810172844 \mathrm{E}-12$ \\
\hline 0.4 & $8.754586142 \mathrm{E}-10$ & $2.3435318188 \mathrm{E}-11$ \\
\hline 0.6 & $1.041634779 \mathrm{E}-8$ & $4.4263789711 \mathrm{E}-11$ \\
\hline 0.8 & $7.438348144 \mathrm{E}-8$ & $2.6707364800 \mathrm{E}-11$ \\
\hline 1 & $1.333333333 \mathrm{E}-7$ & 0 \\
\hline
\end{tabular}




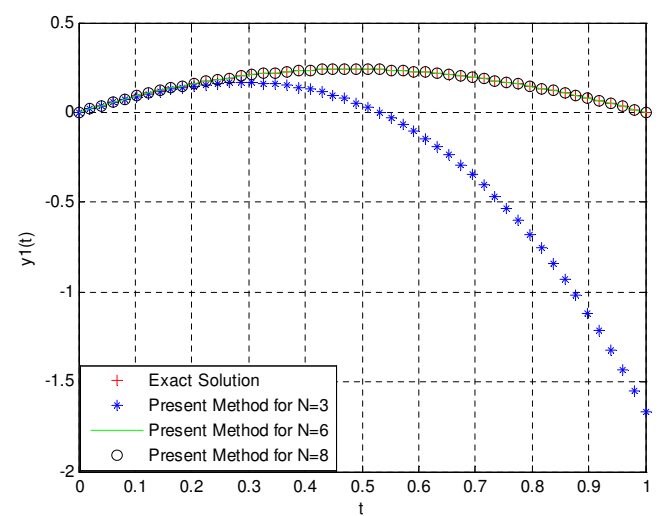

Fig. 1: Numerical and exact solution of Example 2 for $y_{1}(t), N=3,6,8$.

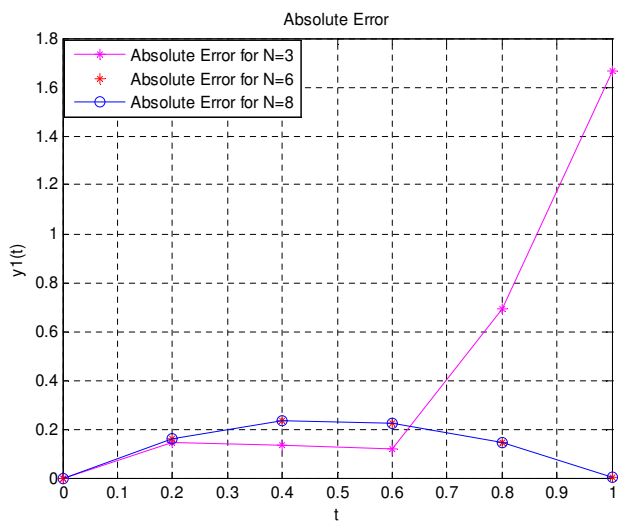

Fig. 3: Comparison of absolute error of Example 2 for $y_{1}(t), N=3,6,8$.

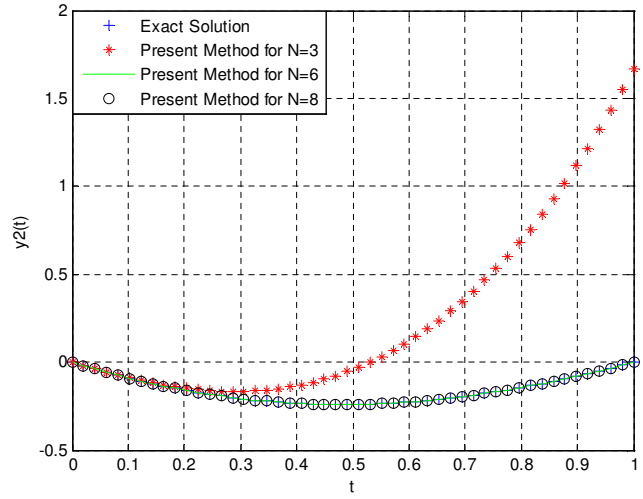

Fig. 2: Numerical and exact solution of Example 2 for $y_{2}(t), N=3,6,8$.

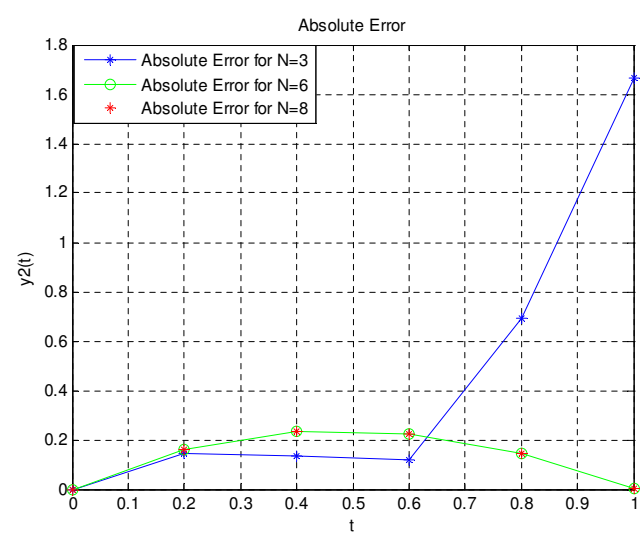

Fig. 4: Comparison of absolute error of Example 2 for $y_{2}(t), N=3,6,8$.

\section{Conclusion}

In this study, a new Fermat matrix collocation method is proposed for nonlinear system of second order boundary value problems. It is observed from Figures and Tables that the method is a simple and powerful tool to obtain the approximate solution. Thus, if $N$ is increased, it can be seen that approximate solutions obtained by the mentioned method are close to the exact solutions. One of the considerable advantages of the method is finding the approximate solutions very easily by using the computer program. Shorter computation time and lower operation count results in a reduction of cumulative truncation errors and improvement of overall accuracy. In addition, the method can also be extended to other models in the future.

\section{References}

[1] Geng F, Cui M (2007) Solving a nonlinear system of second order boundary value problems.J. Math. Anal. Appl.327, 1167-1181. 
[2] Lu J (2007) Variational iteration method for solving a nonlinear system of second-order boundary value problems.Comput. and Math. with Appl.54, 1133-1138.

[3] Dehghan M, Saadatmandi A (2007) The numerical solution of a nonlinear system of second-order boundary value problems using the sinc-collocation method. Math. Comput. Modelling46, 1434-1441.

[4] Saadatmandi A, Farsangi JA (2007) Chebyshev finite difference method for a nonlinear system of second-order boundary value problems.Appl. Math. Comput.192(2), 586-591.

[5] Dehghan M, Lakestani M (2008) Numerical solution of nonlinear system of second-order boundary value problems using cubic Bspline scaling functions.Inter. Journal of Comput. Math.85(9), 1455-1461.

[6] Saadatmandi A, Dehghan M, Eftekhari A (2009) Application of He's homotopy perturbation method for non-linear system of second-order boundary value problems. Nonlinear Analy: Real World Appl.10, 1912-1922.

[7] Chen SH, Hu J, Chen L, Wang CP (2005) Existence results for n-point boundary value problem of second order ordinary differential equations. J. Comput. Appl. Math.180, 425-432.

[8] Cheng XY, Zhong CK (2005) Existence of positive solutions for a second-order ordinary differential system. J. Math. Anal. Appl.312, 14-23.

[9] Lomtatidze A, Malaguti L (2003) On a two-point boundary value problem for the second order ordinary differential equations with singularities.Nonlinear Anal.52, 1553-1567.

[10] Mawhin J, Tisdell C (2003) A note on the uniqueness of solutions to nonlinear, discrete, vector boundary value problems. Nonlinear Anal. Appl.1-2, 789-798.

[11] Thompson HB, Tisdell C (2000) Systems of difference equations associated with boundary value problems for second order systems of ordinary differential equations. J. Math. Anal. Appl.248, 333-347.

[12] Thompson HB, Tisdell C (2002) Boundary value problems for systems of difference equations associated with systems of secondorder ordinary differential equations. Appl. Math. Lett.15(6), 761-766.

[13] Thompson HB, Tisdell C (2003) The nonexistence of spurious solutions to discrete, two-point boundary value problems. Appl.Math. Lett.16(1) 79-84.

[14] Kajani MT, Tabatabaei FG, Maleki M (2012) Rational second kind Chebyshev approximation for solving some physical problems on semi-infinite intervals. Kuwait Journal of Science39(2A), 15-29.

[15] Sezer M (1994) Taylor polynomial solutions of volterra integral equations. Int. J. Math. Educ. Sci. Technol.25(5), 625-633.

[16] Akyüz-Daşcioğlu A, Yaslan HC (2011) The solution of high-order nonlinear ordinary differential equations by Chebyshev series. Appl. Math. Comput.217(12), 5658-5666.

[17] Işık OR, Sezer M, Güney Z (2011) A rational approximation based on Bernstein polynomials for high order initial and boundary values problems. Appl. Math. Comput.217(22), 9438-9450.

[18] Yüzbaşı Ş, Sezer M, Kemancı B (2013) Numerical solutions of integro-differential equations and application of a population model with an improved Legendre method. Appl. Math. Model.37(4), 2086-2101.

[19] Yüzbaşı Ş (2012) On the solutions of a system of linear retarded and advanced differential equations by the bessel collocation approximation. Comput. and Math. with Appl.63(10)1442-1455.

[20] Gökmen E, Sezer M (2013) Taylor collocation method for systems of high-order linear differential-difference equations with variable coefficients. Ain Shams Engineering Journal4,117-125.

[21] Taştekin D, Yalçınbaş S (2014) Fermat Collocation Method for Solvıng a Class of the First Order Nonlinear Differential Equations. Journal of Mathematical Sciences and Applications2(1), 4-9.

[22] Yalçınbaş S, Taştekin D (2014) Fermat Collocation Method for Solvıng a Class of the Second Order Nonlinear Differential Equations. Applied Mathematics and Physics2(2), 33-39.

[23] Gökmen E, Sezer M (2013) Taylor Collocation Method for Nonlinear System of Second Order Boundary Value Problems.Düzce University Journal of Science \& Technology1, 11-23. 